\title{
A Nonsense Polymorphism (Y319X) of the Solute Carrier Family 6 Member 18 (SLC6A18) Gene is Not Associated with Hypertension and Blood Pressure in Japanese
}

\author{
Bita Eslami, Masato Kinboshi, Sumiko Inoue, Kouji Harada, Kayoko Inoue \\ and AкIо KoIzumi \\ Department of Health and Environmental Sciences, Kyoto University Graduate \\ School of Medicine, Kyoto, Japan
}

Eslami, B., Kinboshi, M., Inoue, S., Harada, K., Inoue, K. and Koizumi, A. A Nonsense Polymorphism (Y319X) of the Solute Carrier Family 6 Member 18 (SLC6A18) Gene is Not Associated with Hypertension and Blood Pressure in Japanese. Tohoku J. Exp. Med., 2006, 208 (1), 25-31 — We investigated the possible association of solute carrier family 6 member 18 (SLC6A18) with hypertension and blood pressure in Japanese, since the homologous murine $X T 2$ gene was recently reported to be associated with hypertension. The entire coding region of SLC6A18 was sequenced in 30 unrelated Japanese subjects. The deleterious effects of the observed nonsynonymous single nucleotide polymorphisms (SNPs) on the phenotype were predicted using bioinformatics software. We tested the associations of one deleterious SNP (Y319X) with blood pressure and hypertension in a general population of 1,004 subjects in one area of Japan. Both quantitative and qualitative analyses adjusting for age and body mass index (BMI) as covariates were undertaken. Four synonymous (P7P, T32T, G37G and V387V), three missense (S12C, I32T and L478P) and one nonsense (Y319X: g1230757 C > G) polymorphisms were found. One of the synonymous polymorphisms was novel $(\mathrm{V} 387 \mathrm{~V})$ by reference to the dbSNP database. The Y319X genotype distribution of CC:CG:GG in this population showed frequencies of $0.382,0.461$ and 0.156 , respectively, which followed Hardy-Weinberg equilibrium. The nonsense polymorphism had odds ratios of 0.83 (confidence interval $[\mathrm{CI}]=0.59-1.15, p=$ $0.26)$ in males and $0.96(\mathrm{CI}=0.72-1.29, p=0.80)$ in females with hypertension or current medication for hypertension. For the quantitative analysis, we excluded the current medication subgroup. The nonsense allele was not a significant predictor for systolic or diastolic blood pressure. This is the first report showing that a single polymorphism in SLC6A18 is not associated with hypertension or blood pressure in Japanese. —— hypertension; polymorphism; population science; genetics-human; SLC6A18

(C) 2006 Tohoku University Medical Press

Received September 5, 2005; revision accepted for publication October 14, 2005.

Correspondence: Akio Koizumi, M.D., Ph.D., Professor, Department of Health and Environmental Sciences, Kyoto University Graduate School of Medicine, Kyoto 606-8501, Japan.

e-mail: koizumi@pbh.med.kyoto-u.ac.jp

Bita Eslami and Masato Kinboshi contributed equally to this work. 
Hypertension is a complex trait in which genetic and environmental factors contribute interactively. No single genetic variant has emerged from previous linkage or association analyses as being consistently confirmed in different ethnic populations. However, a number of polymorphisms in candidate genes have been found to be associated with differences in blood pressure (Ruppert and Maisch 2003).

A relatively strong linkage was determined for human hypertension in 5p15 (Rice et al. 2000; $\mathrm{Wu}$ et al. 2003). A study using mice of the inbred strain BPH/2 as a rodent model of hypertension revealed a significant linkage on chromosome 13 (Wright et al. 1999). In addition, studies using the Dahl salt-sensitive (S) rat determined that genomic segments on the $\mathrm{S}$ rat genome harbor blood pressure (BP) quantitative trait loci (QTLs) (Garret et al. 2002), one of which is on rat chromosome (RNO) 1 (Yasser et al. 1999). Furthermore, scanning of the Milan hypertensive rat genome also revealed suggestive QTLs for systolic blood pressure (SBP) on chromosome 1 (Zagato et al. 2000). The homologous regions with BP QTL2 on RNO1 lie on parts of mouse chromosome 13 and human chromosome 5.

According to a recent investigation (Quan et al. 2004), disruption of the $X T 2$ gene, which is located on chromosome 13 in mice, causes hypertension. $X T 2$ is predominantly expressed in the kidney and shows $80 \%$ homology with solute carrier family 6 (neurotransmitter transporter) member 18 (SLC6A18), which was mapped on 5p15.33. Human SLC6A18 is also mainly expressed in the kidney (Kleta et al. 2004), although the physiological function of SLC6A18 has not yet been clarified. Based on these lines of evidence, we hypothesized that the SLC6A18 gene may be a candidate gene for hypertension in humans.

In the present study, we tested the possible contributions of SLC6A18 to blood pressure and hypertension in humans.

\section{Materials and Methods}

Study subjects and study design

In 1998, we established a DNA sample bank, in which 3,434 residents in Noshiro city in the Akita prefecture of Japan donated a blood sample and provided health checkup data for genetic analysis. The data included laboratory test results, a physical examination including height, body weight, blood pressure and electrocardiography, family history, current medications and lifestyle. The blood pressure was measured in the sitting position using a mercury sphygmomanometer after the subject had been seated quietly for more than 5 minutes. SBP and diastolic blood pressure (DBP) were recorded as the means of two measurements taken by public nurses. The criteria for hypertension were SBP $\geqq 140$ or DBP $\geqq 90$ based on the Joint National Committee 7 (JNC7) Classification (Chobanian et al. 2003). Public nurses measured the height and body weight. The body mass index (BMI) was calculated by dividing the body weight (in kilograms) by the square of height (in meters).

First, we selected 30 unrelated DNA samples from the bank by simple randomization in order to identify single nucleotide polymorphisms (SNPs) in the entire coding region of $S L C 6 A 18$. This number results in a power of $>95 \%$ for detecting all variants with frequencies higher than 5\% (Johnson et al. 2001). Next, we tested the associations of the identified SNPs with blood pressure or hypertension by selecting the DNAs of another 1,004 subjects from the bank by simple randomization. The Ethics Committee at Kyoto University approved the present study. Appropriate written informed consent was obtained from all participants.

Extraction, amplification and sequencing of genomic DNA

Genomic DNA was extracted from blood samples using a QIAamp Blood Mini Kit (Qiagen, Hilden, Germany). In order to amplify exons 1-12 (coding region) of $S L C 6 A 18$, polymerase chain reaction (PCR) was performed on $50 \mathrm{ng}$ of genomic DNA as a template using a LA PCR kit Ver. 2.1 (Takara Bio, Tokyo). The PCR products were electrophoresed and purified using a QIAquick Gel Extraction Kit (Qiagen), followed by sequencing with an ABI 3100 Avant DNA sequencer (Applied Biosystems, Foster City, CA, USA). All the detected SNPs were checked using the dbSNP database as a reference. 
Prediction of the effects of the detected SNPs on the function of SLC6A18

We conducted functional analyses for the identified nonsynonymous SNPs using the Polyphen software (http://tux.exbl-heidelberg.de/polyphen/). The protein structure was predicted by SOSUI (http://sosui.proteome. bio.tuat.ac.jp/sosuiframe0.html) and all the detected SNPs were allocated on it.

\section{PCR-restriction fragment length polymorphisms}

PCR-restriction fragment length polymorphisms (RFLP) was conducted for the deleterious SNPs in exon 7 using the primers 5'-ATCTGAGGCCATTCTGAGCTC and 5'-ACAGAAATGAAATGTGCCCACG, which was 414 bp product in length. The PCR products were digested with restriction enzymes, and the allelic-specific PCR-RFLP products were separated by electrophoresis.

\section{Statistical analysis}

The statistical significances of differences between variables were tested by Student's $t$-test and ANOVA when appropriate. Comparisons of frequencies and tests for Hardy-Weinberg equilibrium were conducted using $\chi^{2}$-tests. These analyses were carried out using SAS software (Version 8.2; SAS Institute Inc., Cary, NC, USA). In order to estimate the effects of the genotypes with adjustment for covariates such as age and BMI, multiple regression analysis was conducted using the THESIAS software (http://www.genecanvas.org) (Tregouet et al. 2002). For regression analysis by SAS, three numerical coding schemes were used to represent the genotypes: (1) ( $\mathrm{AA}, \mathrm{AB}, \mathrm{BB})=(0,1,1)$, (2) $(\mathrm{AA}, \mathrm{AB}, \mathrm{BB})=(0,0,1)$ and $(3)(\mathrm{AA}, \mathrm{AB}, \mathrm{BB})=(0,0.5,1)$. The first scheme assumes that allele $B$ is dominant, the second scheme assumes that allele B is recessive and the third scheme assumes a continuous mode.

We estimated the statistical power from the number of subjects for the association studies. Specifically, the statistical power was predicted by the Genetic Power calculator (Purcell et al. 2003) to be $82 \%$ when the $\alpha$ level was set to 0.05 . For this calculation, some assumptions about the genotype relative risk $(\mathrm{AA}=1.6, \mathrm{Aa}=1.01)$, risk allele frequency $(0.4)$, prevalence $(0.25)$ and marker allele frequency $(0.4)$ were made.

\section{Results}

We found four synonymous (P7P, T32T, G37G and V387V), three nonsynonymous (S12C, I32T and L478P) and one nonsense (Y319X: g1230757 C > G) polymorphisms (Table 1) in the entire coding region of SLC6A18 in the first 30 DNA samples. One of the synonymous polymorphisms was novel (V387V) by reference to the dbSNP database. The Polyphen software predicted that the three nonsynonymous variants were not deleterious. Therefore, we only tested the nonsense variant, Y319X, for its associations with blood pressure and hypertension in 1,004 randomly selected subjects. This polymorphism (Y319X) causes a substitution $(\mathrm{C}>\mathrm{G})$ in exon 7 , which introduces a stop codon at residue 319 , thereby truncating about half the 629 amino acids of the protein (Fig. 1).

The demographic characteristics of the 1,004 subjects and their subgroups are shown in Table 2. Among the total subjects, the Y319X genotype distributions of $\mathrm{CC}: \mathrm{CG}: \mathrm{GG}$ showed frequencies

TABLE 1. Single nucleotide polymorphisms (SNPs) identified in the coding region of SLC6A18 in 30 Japanese

\begin{tabular}{cccc}
\hline Exon & Genome position & SNP & Allele frequency $(\%)$ \\
\hline 1 & $\mathrm{~g} 1215613$ & $\mathrm{P} 7 \mathrm{P}(\mathrm{G}>\mathrm{A})$ & $\mathrm{G}: 28(46.7 \%) / \mathrm{A}: 32(53.3 \%)$ \\
1 & $\mathrm{~g} 1215626$ & $\mathrm{~S} 12 \mathrm{C}(\mathrm{T}>\mathrm{A})$ & $\mathrm{T}: 28(46.7 \%) / \mathrm{A}: 32(53.3 \%)$ \\
1 & $\mathrm{~g} 1215687$ & $\mathrm{I} 32 \mathrm{~T}(\mathrm{C}>\mathrm{T})$ & $\mathrm{C}: 18(30.0 \%) / \mathrm{T}: 42(70.0 \%)$ \\
1 & $\mathrm{~g} 1215688$ & $\mathrm{~T} 32 \mathrm{~T}(\mathrm{~T}>\mathrm{C})$ & $\mathrm{T}: 30(50.0 \%) / \mathrm{C}: 30(50.0 \%)$ \\
1 & $\mathrm{~g} 1215703$ & $\mathrm{G} 37 \mathrm{G}(\mathrm{A}>\mathrm{G})$ & $\mathrm{A}: 18(30.0 \%) / \mathrm{G}: 42(70.0 \%)$ \\
7 & $\mathrm{~g} 1230757$ & $\mathrm{Y} 319 \mathrm{X}(\mathrm{C}>\mathrm{G})$ & $\mathrm{C}: 32(53.3 \%) / \mathrm{G}: 28(46.7 \%)$ \\
9 & $\mathrm{~g} 1233699$ & $\mathrm{~V} 387 \mathrm{C}(\mathrm{C}>\mathrm{T})$ & $\mathrm{C}: 50(83.3 \%) / \mathrm{T}: 10(16.7 \%)$ \\
10 & $\mathrm{~g} 1234465$ & $\mathrm{~L} 478 \mathrm{P}(\mathrm{C}>\mathrm{T})$ & $\mathrm{C}: 42(70.0 \%) / \mathrm{T}: 18(30.0 \%)$ \\
\hline
\end{tabular}




\section{Outside}

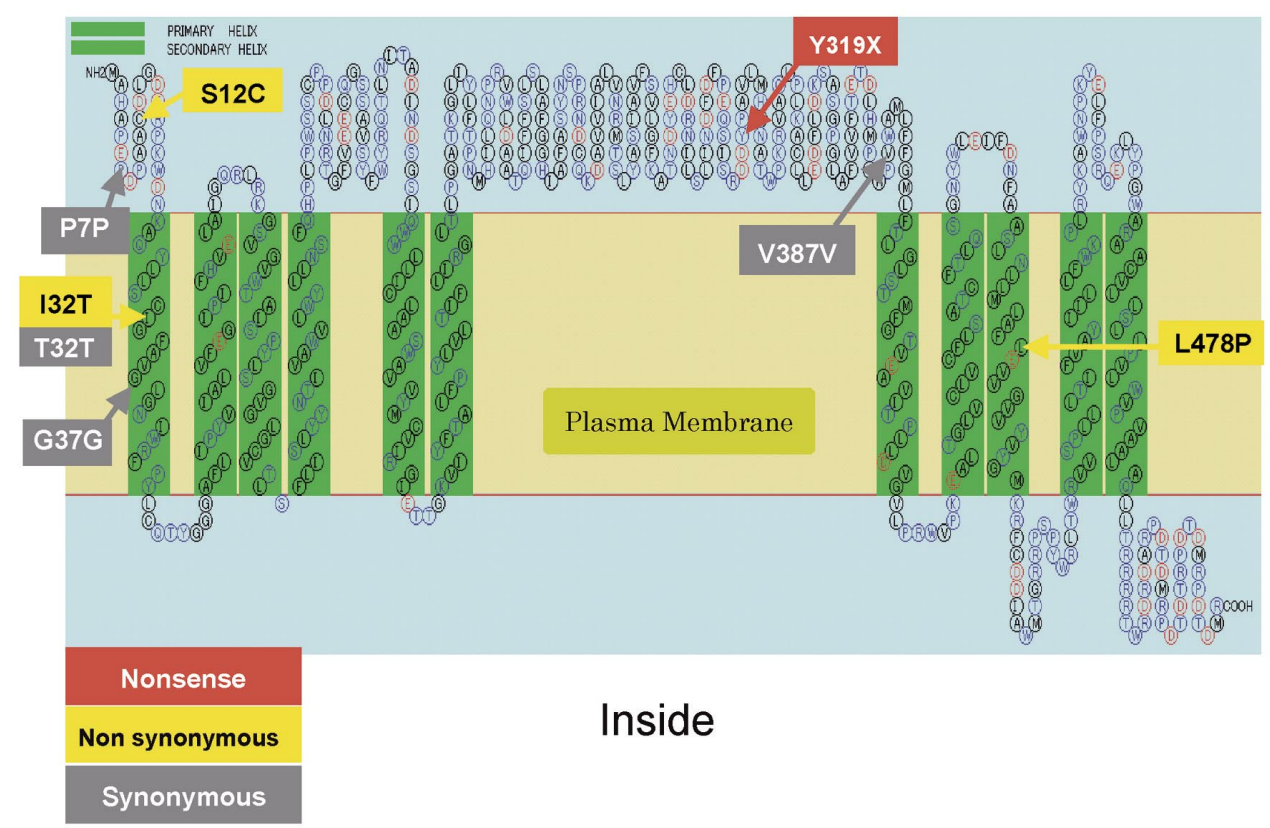

Fig. 1. Allocations of the SNPs on the predicted structure of SLC6A18.

TABLE 2. Characteristics of the total subjects

\begin{tabular}{lccc}
\hline & Total & Males & Females \\
\hline No. & 1,004 & 437 & 567 \\
Age $($ years $)$ & $51.6 \pm 13.5$ & $49.7 \pm 14.3$ & $53.1 \pm 12.6^{*}$ \\
BMI $\left(\mathrm{kg} / \mathrm{m}^{2}\right)$ & $23.3 \pm 3.1$ & $23.5 \pm 3.0$ & $23.2 \pm 3.1$ \\
Total cholesterol $(\mathrm{mg} / 100 \mathrm{ml})$ & $203 \pm 35.0$ & $199 \pm 34.7$ & $206 \pm 35.0^{*}$ \\
HDL $(\mathrm{mg} / 100 \mathrm{ml})$ & $55.6 \pm 14.0$ & $52.1 \pm 13.7$ & $58.3 \pm 13.6^{*}$ \\
Blood glucose $(\mathrm{mg} / 100 \mathrm{ml})$ & $97.5 \pm 18.8$ & $101.7 \pm 22.2$ & $94.2 \pm 15.0^{*}$ \\
Smoking ${ }^{1}(\%)^{* *}$ & & & \\
$\quad$ Non & $612(61.0 \%)$ & $101(23.1 \%)$ & $511(90.1 \%)$ \\
$\quad$ Former & $128(12.7 \%)$ & $104(23.8 \%)$ & $24(4.2 \%)$ \\
$\quad$ Current & $264(26.3 \%)$ & $232(53.1 \%)$ & $32(5.6 \%)$ \\
Blood pressure $(\mathrm{mmHg})$ & $127 \pm 18.7$ & $130 \pm 17.8$ & $125 \pm 19.2^{*}$ \\
$\quad$ Systolic & $78.2 \pm 11.6$ & $80.7 \pm 11.3$ & $76.3 \pm 11.5{ }^{*}$ \\
$\quad$ Diastolic & $268(26.7 \%)$ & $133(30.4 \%)$ & $135(23.8 \%)$ \\
Hypertension & & & \\
Current antihypertensive & $182(18.1 \%)$ & $79(18.1 \%)$ & $103(18.2 \%)$ \\
medication & $334(33.3 \%)$ & $126(28.8 \%)$ & $208(36.7 \%)^{*}$ \\
Family history & & & \\
\hline
\end{tabular}

Values are the mean \pm S.D.

HDL, high-density lipoprotein; BMI, body mass index.

${ }^{1}$ Non, nonsmoker; Former, former smoker; Current, current smoker.

${ }^{*} p<0.05$ between males and females by Student's $t$-test.

${ }^{* *} p<0.05$ between males and females by the $\chi^{2}$-test. 
TABLE 3. Comparison of blood pressure, hypertension and risk factors among genotypes for the nonsense allele (Y319X) in two subgroups

\begin{tabular}{|c|c|c|c|c|c|c|c|c|}
\hline \multirow{2}{*}{$\begin{array}{l}\text { Subgroup } \\
\text { Genotype }\end{array}$} & \multicolumn{4}{|c|}{ Males } & \multicolumn{4}{|c|}{ Females } \\
\hline & $\mathrm{CC}$ & $\mathrm{CG}$ & GG & $p^{*}$ & $\mathrm{CC}$ & $\mathrm{CG}$ & GG & $p^{*}$ \\
\hline No. & 164 & 204 & 69 & & 220 & 259 & 88 & \\
\hline Age & $51.0 \pm 14.8$ & $48.8 \pm 13.3$ & $49.3 \pm 15.6$ & 0.32 & $53.2 \pm 13.1$ & $53.0 \pm 12.4$ & $52.8 \pm 12.4$ & 0.97 \\
\hline BMI $\left(\mathrm{kg} / \mathrm{m}^{2}\right)$ & $23.3 \pm 2.8$ & $23.8 \pm 3.1$ & $23.2 \pm 3.1$ & 0.25 & $23.2 \pm 3.1$ & $23.3 \pm 3.2$ & $22.8 \pm 2.7$ & 0.42 \\
\hline \multicolumn{9}{|c|}{$\begin{array}{l}\text { Hypertension or } \\
\text { current antihypertensive }\end{array}$} \\
\hline medication & $51(31.1 \%)$ & $85(41.7 \%)$ & $23(33.3 \%)$ & 0.09 & $61(27.7 \%)$ & $82(31.7 \%)$ & $21(23.9 \%)$ & 0.33 \\
\hline Family history & $50(30.5 \%)$ & $56(27.5 \%)$ & $20(29.0 \%)$ & 0.81 & $78(35.5 \%)$ & $98(37.8 \%)$ & $32(36.4 \%)$ & 0.86 \\
\hline \multicolumn{9}{|c|}{ Blood pressure ( $\mathrm{mmHg}$ ) excluding those currently taking antihypertensive medications } \\
\hline No. & 139 & 162 & 57 & & 186 & 204 & 74 & \\
\hline Systolic & $126 \pm 14.1$ & $127 \pm 16.4$ & $124 \pm 19.3$ & 0.48 & $122 \pm 16.1$ & $121 \pm 16.0$ & $118 \pm 17.2$ & 0.21 \\
\hline Diastolic & $78.3 \pm 9.4$ & $79.1 \pm 10.1$ & $77.2 \pm 12.6$ & 0.47 & $74.3 \pm 10.0$ & $73.9 \pm 10.1$ & $72.1 \pm 11.5$ & 0.29 \\
\hline
\end{tabular}

Values are the mean \pm S.D.

BMI, body mass index.

* $p$ refers to ANOVA for age, BMI, blood pressure, hypertension or past history and family history.

TABLE 4. Effects of the nonsense SNP (Y319X) on blood pressure and hypertension, as evaluated by multiple regression analyses using THESIAS in two subgroups ${ }^{1}$

\begin{tabular}{|c|c|c|c|c|c|c|}
\hline & \multicolumn{3}{|c|}{ Males } & \multicolumn{3}{|c|}{ Females } \\
\hline & $\begin{array}{l}\text { Nonsense allele } \\
\qquad(\mathrm{C}>\mathrm{G})\end{array}$ & Age & BMI & $\begin{array}{l}\text { Nonsense allele } \\
\qquad(\mathrm{C}>\mathrm{G})\end{array}$ & Age & BMI \\
\hline \multicolumn{7}{|c|}{ Phenotype } \\
\hline \multicolumn{7}{|l|}{ SBP } \\
\hline Slope & -0.36 & 0.24 & 1.39 & -1.24 & 0.23 & 1.73 \\
\hline CI & $(-2.57-1.85)$ & $(0.12-0.36)$ & $(0.79-1.99)$ & $(-3.08-0.60)$ & $(0.11-0.34)$ & $(1.30-2.16)$ \\
\hline$p$ & 0.75 & 0.000081 & 0.000006 & 0.19 & 0.000096 & $<0.000001$ \\
\hline \multicolumn{7}{|l|}{ DBP } \\
\hline Slope & -0.07 & 0.15 & 0.95 & -0.75 & 0.06 & 1.09 \\
\hline CI & $(-1.44-1.30)$ & $(0.08-0.23)$ & $(0.59-1.31)$ & $(-1.92-0.43)$ & $(-0.01-0.13)$ & $(0.80-1.38)$ \\
\hline$p$ & 0.92 & 0.000026 & $<0.000001$ & 0.21 & 0.11 & $<0.000001$ \\
\hline \multicolumn{7}{|c|}{ Hypertension } \\
\hline OR & 0.83 & 1.00 & 1.03 & 0.96 & 1.02 & 0.95 \\
\hline $\mathrm{CI}$ & $(0.59-1.15)$ & $(0.99-1.02)$ & $(0.96-1.12)$ & $(0.72-1.29)$ & $(1.00-1.03)$ & $(0.89-1.03)$ \\
\hline$p$ & 0.26 & 0.77 & 0.38 & 0.80 & 0.08 & 0.21 \\
\hline
\end{tabular}

SBP, systolic blood pressure; DBP, diastolic blood pressure, OR, odds ratio; CI, confidence interval;

BMI, body mass index.

${ }^{1}$ Subjects currently taking antihypertensive medications were excluded from the analysis. 
of $0.382,0.461$ and 0.156 , respectively, which followed Hardy-Weinberg equilibrium. Comparisons of covariates such as age, BMI, hypertension, family history and blood pressure without antihypertensive medication among the wild-type (CC), heterozygous (CG) and homozygous (GG) genotypes were conducted in each subgroup.

In the following analysis, subjects with current antihypertensive medication were excluded from the quantitative analysis to eliminate the effects of medication on blood pressure. As shown in Table 3 , there were no significant differences in blood pressure, prevalence and family history of hypertension and risk factors among the three genotypes.

In addition, other covariates such as total cholesterol, high-density lipoprotein (HDL), blood glucose and smoking status were similar among the three genotypes within the two subgroups (data not shown).

The results of testing the associations of the polymorphism with SBP, DBP and hypertension in the two subgroups by THESIAS are shown in Table 4. The nonsense polymorphism had relative risks (odds ratios) of 0.83 (confidence interval $[\mathrm{CI}]=0.59-1.15, p=0.26$ ) in males and 0.96 $(\mathrm{CI}=0.72-1.29, p=0.80)$ in females, suggesting that this SNP did not contribute to hypertension in either of the two subgroups. The regression analysis by the SAS software also showed no significant differences in the dominant, recessive and continuous genotypes in the total population and the two subgroups (data not shown).

\section{Discussion}

Hypertension is a common phenotype that is considered to be a multifactorial trait. In addition, genetic factors (such as the $A G T$ gene) (Fardella et al. 1999) are considered to elevate or decrease blood pressure in concert with environmental factors or other biological factors such as sex, age and BMI (Hamann and Sharma 2002; WHO 2002).

The $X T 2$ gene in mice shows $80 \%$ homology with human SLC6A18, and mediates sodiumdependent high-affinity reuptake of glycine on the brush border membrane of renal proximal tubules (Quan et al. 2004). However, XT2 does not increase the plasma membrane glycine uptake activity in cultured cells, suggesting that it may require the coexpression of other co-transporter or accessory proteins to exhibit its full activity (Quan et al. 2004). If these co-transporter or accessory proteins are deficient in humans, SLC6A18 cannot express its full function in the kidneys. Finally, SLC6A18 seems to have little, if any, physiologically significant function, since a large majority of individuals have a stop codon. Whatever the mechanisms may be, it is likely that haploinsufficiency or loss of function of the SLC6A18 transporter does not cause hypertension in humans.

The present study failed to show that a single nonsense polymorphism in SLC6A18 is involved in either quantitative blood pressure or qualitative hypertension in the general population in Japan. The data presented in the current study argue against an association between hypertension and SLC6A18 in humans. Although a previous animal study by Quan et al. (2004) confirmed that the $X T 2$ gene is associated with hypertension in mice, our present data in humans failed to confirm an association between SLC6A18 and hypertension. Therefore, the previous association found in mice may not be generally applicable to hypertension in humans.

The present results are limited to the current study population. Therefore, in order to definitively assess the role of this candidate gene in humans, additional investigations in other populations are required. Another limitation of the present study is the statistical power. We assumed that the genotype relative risk was high. However, since hypertension is a multifactorial phenotype influenced by many genetic factors, it may be better to assume a lower genotype relative risk. In this case, we would have to increase the sample size.

\section{Acknowledgments}

We are grateful to Chieko Ishiguro for advice regarding this study. We also thank Mr. Benjamin Seery for critical reading of the manuscript. 


\section{References}

Chobanian, A.V., Bakris, G.L., Black, H.R., Cushman, W.C., Green, L.A., Izzo, J.L., Jr., Jones, D.W., Materson, B.J., Oparil, S., Wright, J.T., Jr. \& Roccella, E.J. (2003) The Seventh Report of the Joint National Committee on Prevention, Detection, Evaluation, and Treatment of High Blood Pressure: the JNC 7 report. JAMA, 289, 2560-2572.

Fardella, C., Zamorano, P. \& Mosso, L. (1999) A(-6)G variant of angiotensinogen gene and aldosterone levels in hypertensives. Hypertension, 34, 779-781.

Garrett, M.R., Joe, B., Dene, H. \& Rapp, J.P. (2002) Identification of blood pressure quantitative trait loci that differentiate two hypertensive strains. J. Hypertens., 20, 2399-2406.

Hamann, A. \& Sharma, A.M. (2002) Genetics of obesity and obesity-related hypertension. Semin. Nephrol., 22, 100-104.

Johnson, G.C., Esposito, L., Barratt, B.J., Smith, A.N., Heward, J., Di genova, G., Ueda, H., Cordell, H.J., Eaves, I.A., Dudbridge, F., Twells, R.C., Payne, F., Hughes, W., Nutland, S., Stevens, H., Carr, P., Tuomilehoto-Wolf, E., Tuomilehto, J., Gough, S.C., Clayton, D.G. \& Todd, J.A. (2001) Haplotype tagging for the identification of common disease genes. Nat. Genet., 29, 233-237.

Kleta, R., Romeo, E., Ristic, Z., Ohura, T., Stuart, C., Arcos-Burgos, M., Dave, M.H., Wagner, C.A., Camargo, S.R., Inoue, S., Matsuura, N., Helip-Wooley, A., Bockenhauer, D., Warth, R., Bernardini, I., Visser, G., Eggermann, T., Lee, P., Chairoungdua, A., Jutabha, P., Babu, E., Nilwarangkoon, S., Anzai, N., Kanai, Y., Verrey, F., Gahl, W.A. \& Koizumi, A. (2004) Mutations in SLC6A19, encoding B ${ }^{0}$ AT1, cause Hartnup disorder. Nat. Genet., 36, 999-1002.

Purcell, S., Cherny, S.S. \& Sham, P.C. (2003) Genetic Power Calculator: design of linkage and association genetic mapping studies of complex Traits. Bioinformatics, 19,
149-150.

Quan, H., Athirakul, K., Wetsel, W.C,, Torres, G.E., Stevens, R., Chen, Y.T., Coffman, T.M. \& Caron, M.G. (2004) Hypertension and impaired glycine handling in mice lacking the orphan transporter XT2. Mol. Cell Biol., 24, 4166-4173.

Rice, T., Rankinen, T., Province, M.A., Chagnon, Y.C., Perusse, L., Borecki, I.B, Bouchard, C. \& Rao, D.C. (2000) Genome-wide linkage analysis of systolic and diastolic blood pressure: the Quebec Family Study. Circulation, 102, 1956-1963.

Ruppert, V. \& Maisch, B. (2003) Genetics of human hypertension. Herz, 28, 655-662.

Tregouet, D.A., Barbaux, S., Escolano, S., Tahri, N., Golmard, J.L., Tiret, L. \& Cambien, F. (2002) Specific haplotypes of the P-selectin gene are associated with myocardial infarction. Hum. Mol. Genet., 11, 2015-2023.

World Health report 2002: reducing risks, promoting healthy life. Geneva, Switzerland: World Health Organization (2002) Accessed at http://www.who.int/whr/2002/.

Wright, F. A., O'Connor, D. T., Roberts, E., Kutey, G., Berry, C.C., Yoneda, L. U., Timberlake, D. \& Schlager, G. (1999) Genome scan for blood pressure loci in mice. Hypertension, 34, 625-630.

Wu, Z., Nakura, J., Abe, M., Jin, J.J., Yamamoto, M., Chen, Y., Tabar, Y., Yamamoto, Y., Igase, M., Bo, X., Kohara, K. \& Miki, T. (2003) Genome-wide linkage disequilibrium mapping of hypertension in Japan. Hypertens. Res., 26, 533-540.

Yasser, S., Michael, R.G., Soon, J.L., Howard, D. \& John, P.R. (1999) Localization of a blood pressure QTL on rat chromosome 1 using Dahl rat congenic strains. Physiol. Genomics., 1, 119-125.

Zagato, L., Modica, R., Florio, M., Torielli, L., Bihoreau, M.T., Bianchi, G. \& Tripodi, G. (2000) Genetic mapping of blood pressure quantitative trait loci in Milan hypertensive rats. Hypertension, 36, 734-739. 